\title{
Crestal Sinus Elevation for Simultaneous Implant Placement
}

\author{
Arun K Garg ${ }^{1}$, Gregori M Kurtzman², Lanka Mahesh ${ }^{3}$
}

\begin{abstract}
Insufficient crestal height may present in the posterior maxilla that will require osseous grafting to place implants. When sufficient height is present to stabilize the implant at placement, simultaneous sinus augmentation via a crestal approach with implant placement can be performed. The crestal approach when applicable has fewer complications then lateral sinus augmentation procedures and is more comfortable for the patient during the post operative period. This article shall describe the crestal sinus augmentation technique using special reamers that are safe ended to elevate the sinus membrane without potential tearing.

Keywords: Crestal sinus augmentation, Sinus grafting, Summers approach.

International Journal of Oral Implantology and Clinical Research (2018): 10.5005/jp-journals-10012-1182
\end{abstract}

\section{INTRODUCTION}

The posterior maxilla can be a challenge to implant placement related to the maxillary sinus. The sinus may enlarge (pneumatize) related to patient age, sinus issues, and how long the site has been edentulous or related to periodontal bone loss on the tooth that will or has been extracted. ${ }^{1}$ Crestal bone height, hence, may be diminished from the superior direction (enlargement of the sinus) or from the inferior aspect of the ridge (periodontal bone loss). This can complicate implant placement due to insufficient available to house an implant. Short implants have been advocated for these clinical situations but may not be the approach desired or insufficient height may not be present to even place these short implants.

Two approaches have been reported in the literature to the increase crestal bone height so that sufficient bone height may allow implant placement. The lateral sinus augmentation was first reported in 1980 by Boyne related to the pioneering work performed by Tatum. ${ }^{2}$ This technique was complex and required surgical skill with utilization being applied to any crestal height remaining from paper thin to varying residual thicknesses. The other technique was first reported by Summers in 1994 and used a crestal approach to simplify the surgical aspect. This was designed when there was a sufficient height to stabilize the implant at placement, but an additional height was needed to encompass the implant within the bone. ${ }^{3}$

The crestal approach requires sufficient bone height to stabilize the implant as the implant is required in this technique to tent up the sinus membrane and allow the graft to mature into host bone to encompass the apical portion of the implant. It has been suggested that a minimum height of $4 \mathrm{~mm}$ is required to achieve that goal. ${ }^{4,5}$

There is some consensus that a 4-mm increase in height can be achieved with the crestal approach. ${ }^{6,7}$ With other authors stating greater height increases are possible to a possible $10 \mathrm{~mm}$ gain. ${ }^{8}$ When less available initial crestal height presents, a lateral sinus augmentation should be considered a better approach. The crestal approach works well in single sites or two adjacent sites but may not be suitable when more than two adjacent implants are planned and grafting needed at each site. The crestal approach for sinus augmentation has demonstrated a clinical success of over $93 \%$ as reported in the literature. ${ }^{9}$ Typically, multiple adjacent sites will require graft placement medial to where the implants are being placed and elevation of the sinus membrane medially cannot be performed through the crestal osteotomy.
${ }^{1}$ Private Practice, Miami, Florida, USA

${ }^{2}$ Private Practice, Silver Spring, Maryland, USA

${ }^{3}$ Private Practice, New Delhi, India

Corresponding Author: Gregori M Kurtzman, Private Practice, Silver Spring, Maryland, USA, Phone: +13015983500, e-mail: drimplants@ aol.com

How to cite this article: Garg AK, Kurtzman GM, et al. Crestal Sinus Elevation for Simultaneous Implant Placement. Int J Oral Implantol Clin Res 2018;9(1-3):49-54.

Source of support: Nil

Conflict of interest: None

Crestal sinus elevation may be performed in a site that has been edentulous and healed or at the time of extraction when implant stability can be achieved simultaneous with sinus augmentation. The process begins with the evaluation of the radiograph to determine the height of the residual ridge between the crest and the sinus floor. This height is measured with an instrument on the radiographic film or with software when a digital radiograph has been taken. The resulting height measurement will determine how deep the initial drill will be taken to avoid potentially tearing the sinus membrane and it is recommended by the authors that $2 \mathrm{~mm}$ be deducted from the determined height. For example, if the height measures $7 \mathrm{~mm}$, that depth for the pilot drill be set at $5 \mathrm{~mm}$. Traditional osteotomy burs are not suited for sinus elevation procedures as the tip of the drill, although well suited for bone cutting, will tear the sinus membrane when it contacts it. Thus, osteotomy drills are required with safe ends that will bump up the membrane (elevate it) when contact the delicate structure during site preparation. Although safer than traditional drills, these drills should be limited in how high they are taken past the sinus floor to avoid creating tension in the sinus membrane and subsequent tearing with further advancement. Thus, a physical stop on the drill ensures that accidental advancement is not possible due to free-handing drill depth.

\section{Sinus Crestal Approach Kit}

The Sinus Crestal Approach kit (ImplantVision, Miami, FL) contains all the tools required for a crestal sinus elevation from site preparation 


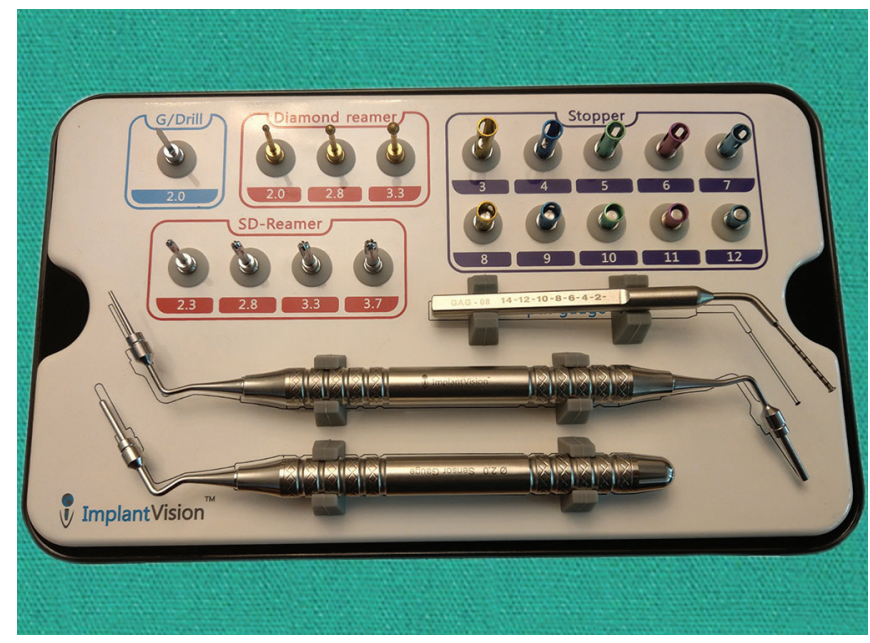

Fig. 1: Crestal sinus kit (ImplantVision) with its components

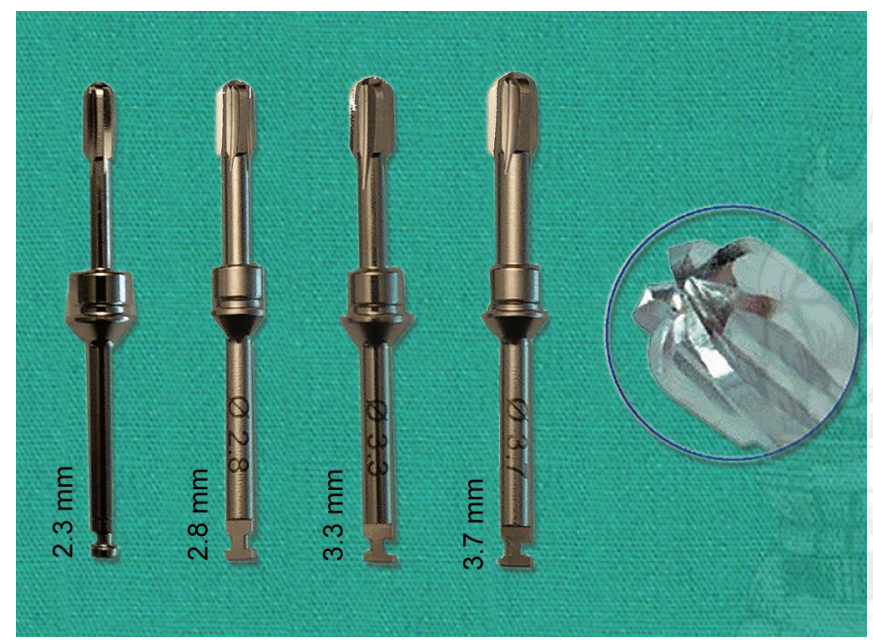

Fig. 3: SD reamers in increasing diameters utilized to penetrate the sinus floor without tearing the sinus membrane due to its rounded safe-end (right)

to graft placement into the elevated sinus prior to implant insertion (Fig. 1). Having all the necessary tools in a single kit allows the practitioner to improve surgical treatment efficiency and have the items easier to access during treatment.

An edentulous site to be treated, the initial osteotomy is made with a pilot drill (guide drill, Fig. 2 left) resulting in a $2 \mathrm{~mm}$ osteotomy. As recommended previously depth should be $2 \mathrm{~mm}$ shy of the measured sinus floor. Depth stoppers can be used on the guide drill to improve depth accuracy. When a thin ridge is present and a starter is desired to prevent jumping of the guide drill with the crest, a diamond reamer may be used. These diamond reamers are a ball diamond available in three diameters (Fig. 2 right). If crestal sinus elevation is to be performed at an immediate extraction site, following tooth extraction, the socket is cleaned of residual soft tissue with the diamond reamers. This eliminates any soft tissue that may hamper osseous contact with the implant surface. The diamond reamers are run in the surgical handpiece at 800-1,200 rpm.

Following initial site preparation, the site diameter is enlarged to less than the diameter of the implant planned for the site. This will allow the implant to exert some osseocompression upon placement and improve initial stability especially in the less dense bone of the posterior maxilla. To achieve this, sinus depth (SD) reamers are utilized (Fig. 3, left). These drills have a safe tip designed to elevate the sinus

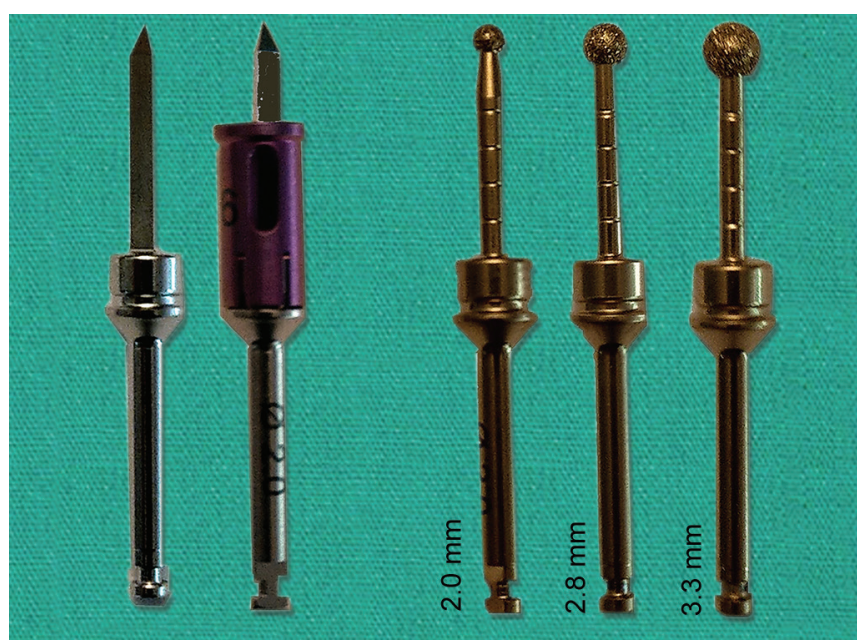

Fig. 2: Guide drill (left) for initial pilot osteotomy in the posterior edentulous maxilla and diamond reamers (right) for removal of residual soft tissue in the extraction site before initiating the crestal sinus lift process

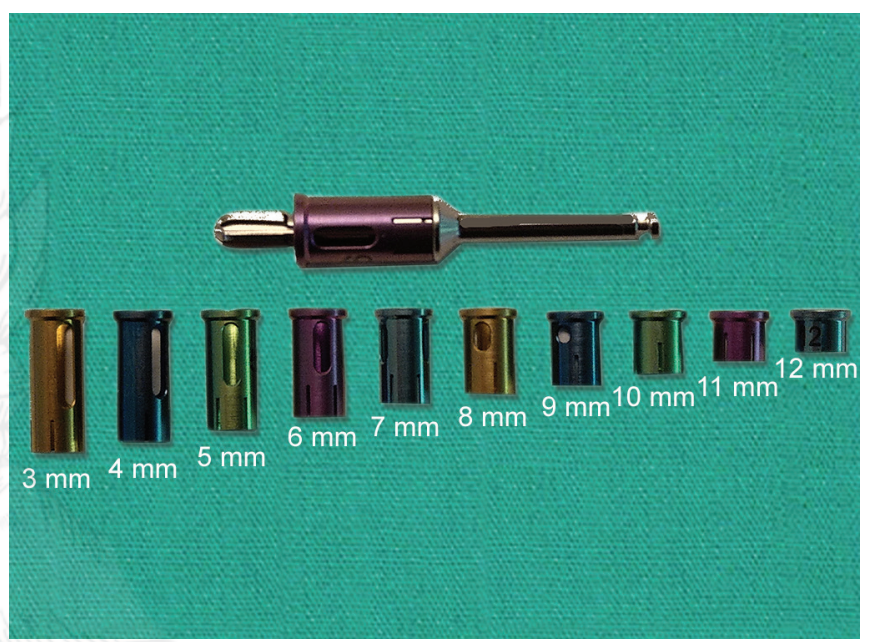

Fig. 4: Stoppers for use with the SD reamers, sensor gauge, bone packer, guide drill, and diamond reamers to guide depth penetration and prevent membrane tearing

upon contact with it and avoid tearing the delicate membrane (Fig. 3 right). Length stoppers are provided in the kit and their designation indicates the depth the drill may enter the bone before the stopper prevents further advancement (Fig. 4). These start at $3 \mathrm{~mm}$ and increase in $1 \mathrm{~mm}$ increments to a maximum of $12 \mathrm{~mm}$. The stoppers may also be used on the guide drill and hand instruments to guide depth when they are used. The SD reamer drill with a stopper may also be used to verify osteotomy depth in relation to the sinus floor by placement off the handpiece into the site and a radiograph taken.

Several hand instruments are provided in the kit. These include a sensor gauge that is used to explore the apical depth of the osteotomy for contact with the sinus membrane (Fig. 5A). The tip of the sensor gauge is domed to prevent inadvertent puncture of the sinus membrane when exploring the osteotomy. Stoppers may be used on the sensor gauge to increase gauging accuracy when desired. A depth gauge instrument with millimeter markings allows the verification of the osteotomy depth and can be used for measurements of film radiographs to determine the crestal height present (Fig. 5B). A bone syringe with a $2.5-\mathrm{mm}$ barrel diameter aids in the placement of graft material into the void created by elevation 


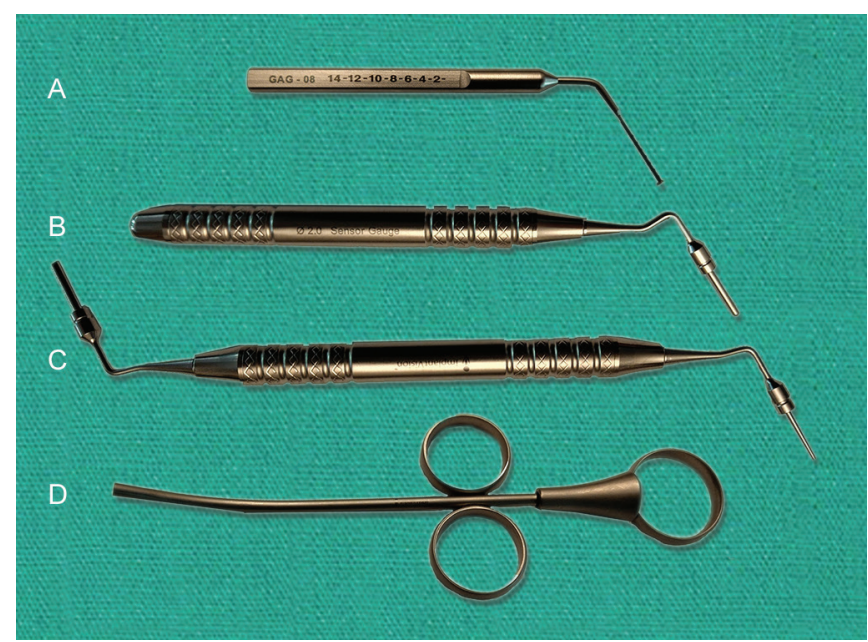

Figs 5A to D: Hand instruments utilized during and after crestal sinus elevation which includes depth gauge: (A) For measurement of the osteotomy depth, sensor gauge; (B) For tactile feel if the membrane has been encountered following the use of the SD reamers, bone packer; (C) For compression of graft material apically into the osteotomy and elevated sinus area and bone syringe; (D) For placement of graft material in the elevated sinus area under the membrane

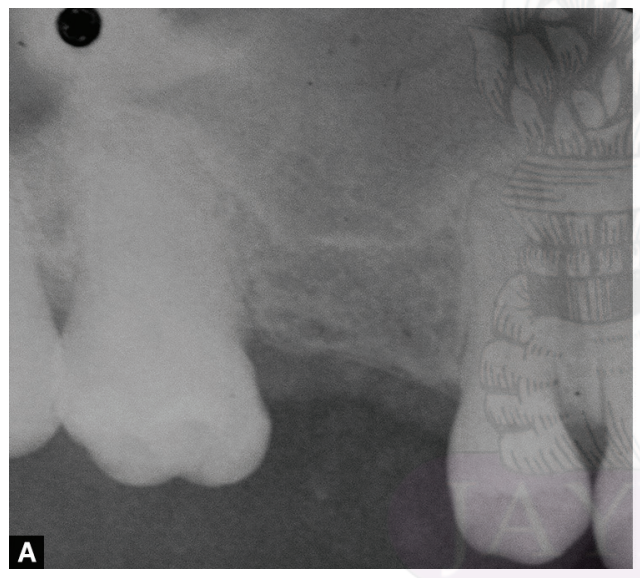

Figs $6 \mathrm{~A}$ and B: Radiograph demonstrating insufficient crestal height to house an implant, but sufficient height for stabilization of an implant at the time of sinus augmentation

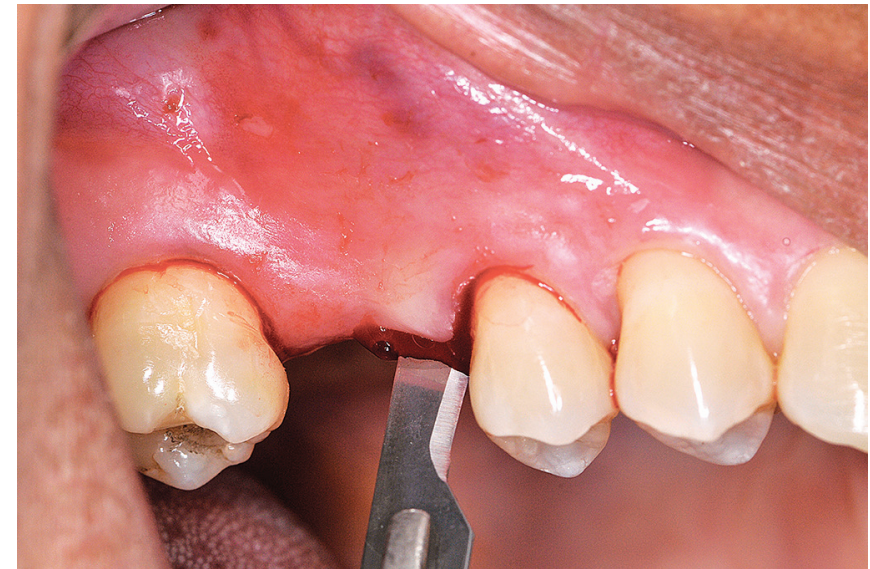

Fig. 7: Crestal incision and sulcular incisions are made with scalpel in the preparation for elevation of a full-thickness envelope flap

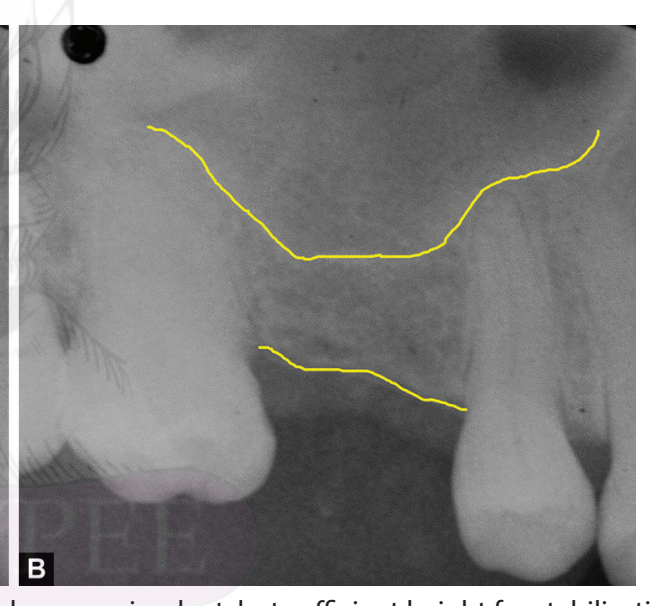

of the sinus membrane (Fig. 5C). A double-ended bone packer with a 1-mm end and a 2.2-mm end that will accommodate the stoppers allows the practitioner to push the graft material apically into the elevated sinus area (Fig. 5D). It is important that sufficient graft is placed into the sinus void under the elevated membrane to ensure that the placement implant upon healing has its apical portion surrounded by the bone.

\section{Case Description}

A 62-year-old female presented with a missing right maxillary molar, stated a desire to replace the missing tooth with an implant. A radiograph was taken and it was determined that with sinus pneumatization, additional height would be needed for implant placement (Fig. 6). As $6 \mathrm{~mm}$ of crestal height was present, initial implant stability would allow a simultaneous crestal sinus lift with implant placement. The patient was scheduled for a crestal sinus augmentation and implant placement.

Local anesthetic was administered and an incision was started in the buccal sulcus of the second molar and continued forward as a crestal incision across the edentulous site (Fig. 7). A full-thickness flap envelop flap was elevated to expose the crest of the ridge (Fig. 8). A 4-mm stopper was placed on the guide drill and the initial pilot hole was created at the center of the edentulous site matching the long axis of the adjacent teeth. A SD reamer of a diameter of $3.7 \mathrm{~mm}$ with a $5-\mathrm{mm}$ stopper was used to increase the osteotomy diameter (Fig. 9). The

(

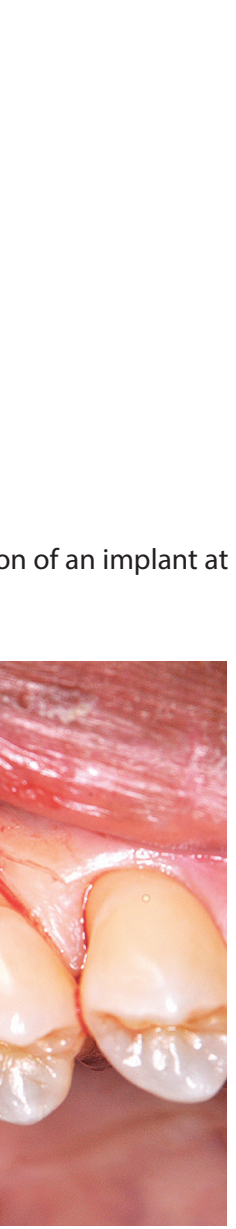

Fig. 8: Envelope flap is elevated to expose the top of the crestal bone at the edentulous site 


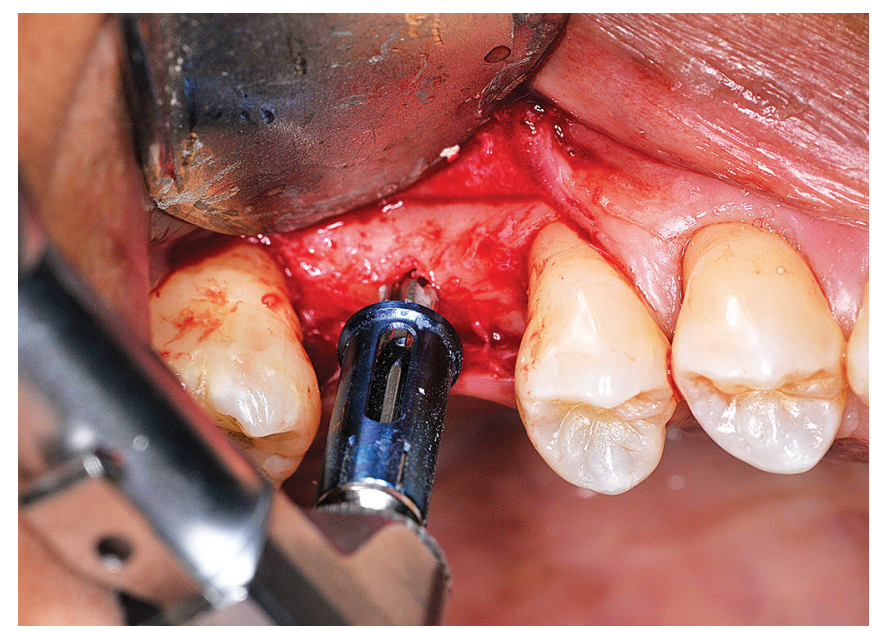

Fig. 9: Pilot hole placement was created with the guide drill $2 \mathrm{~mm}$ short of the measured radiographic height with a stopper utilized to create the initial osteotomy
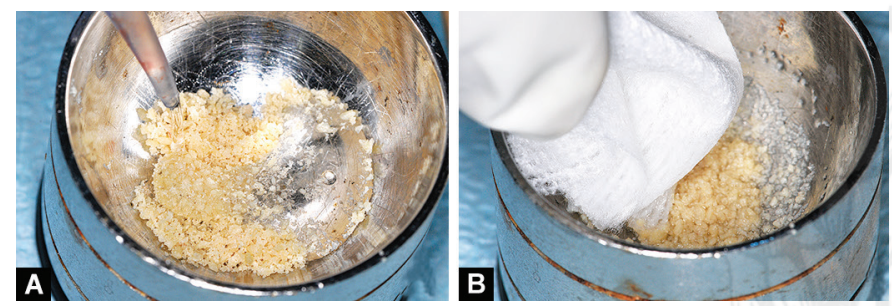

Figs 11A to D: Graft mixture is prepared for placement into the elevated sinus space

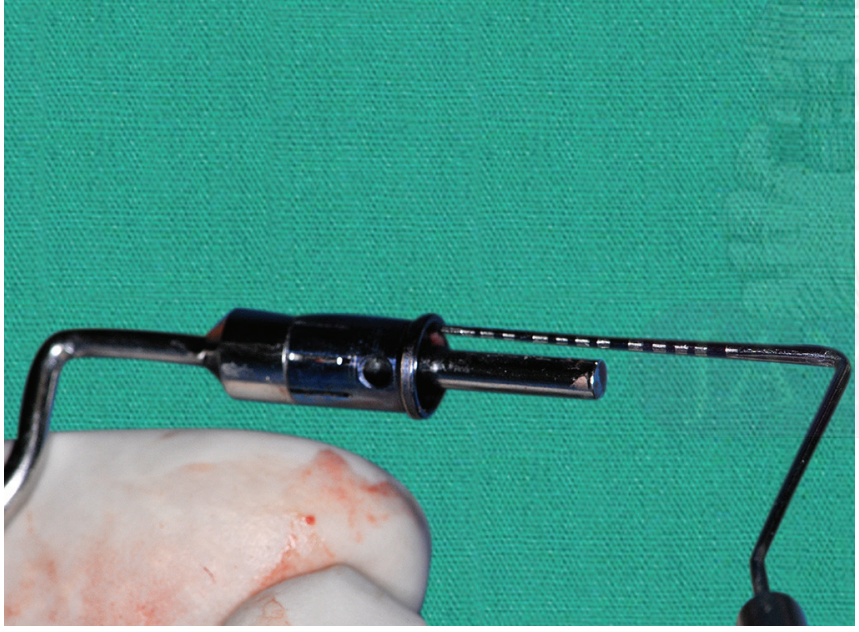

Fig. 12: A $9 \mathrm{~mm}$ stopper is placed on the bone packer instrument to control how deep the instrument would enter the sinus and prevent potential tearing of the membrane

sensor gauge was used to explore the apical aspect of the osteotomy and verify that the sinus membrane had been exposed and the membrane was intact. This was followed up with an 8-mm stopper on the same SD reamer to elevate the exposed sinus membrane (Fig. 10). Again the sensor gauge verified that the membrane was intact.

Freeze-dried cortical allograft graft particles are placed into a sterile dish and flooded with sterile saline (Fig. 11A) to fully rehydrate the allograft material. After 5 minutes, excess saline is wicked from the rehydrated particles with a piece of sterile gauze (Fig. 11B). Carboxymethylcelluous and glycerin are added to the graft particles (Fig. 11C). These materials act as a binder for the graft particles,
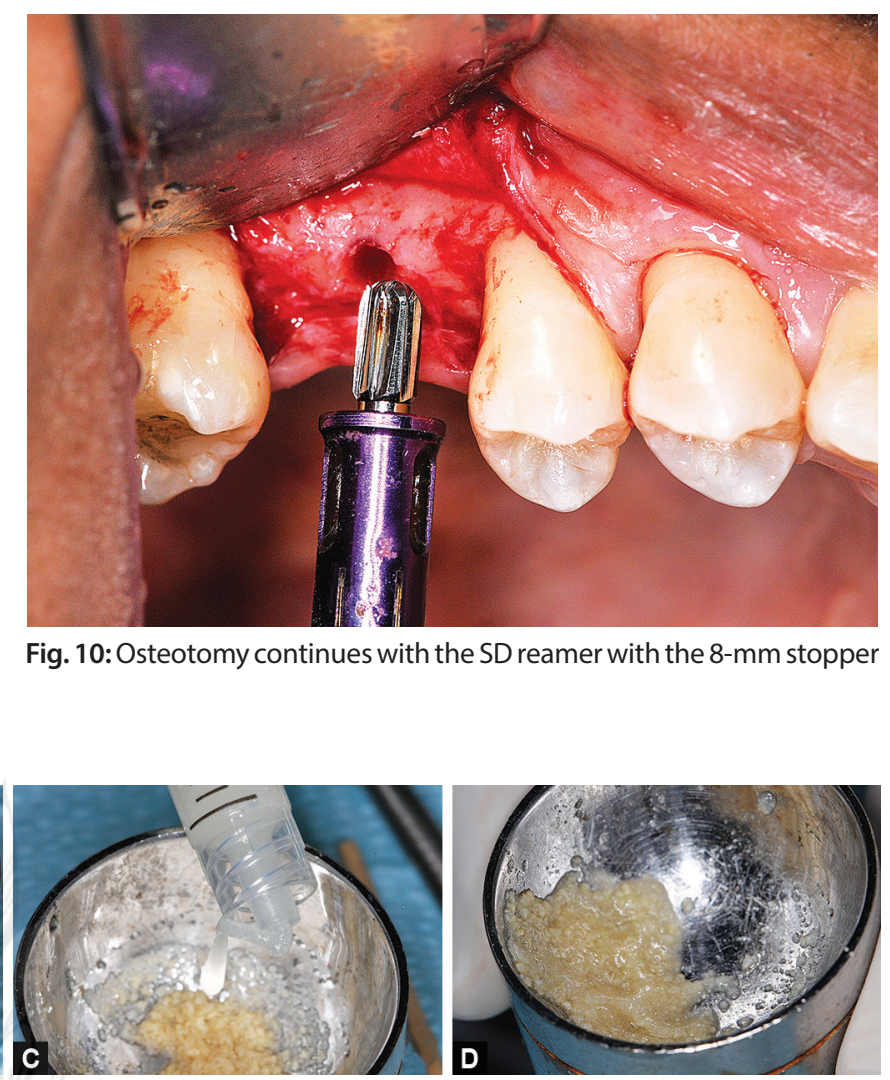

Fig. 10: Osteotomy continues with the SD reamer with the 8-mm stopper

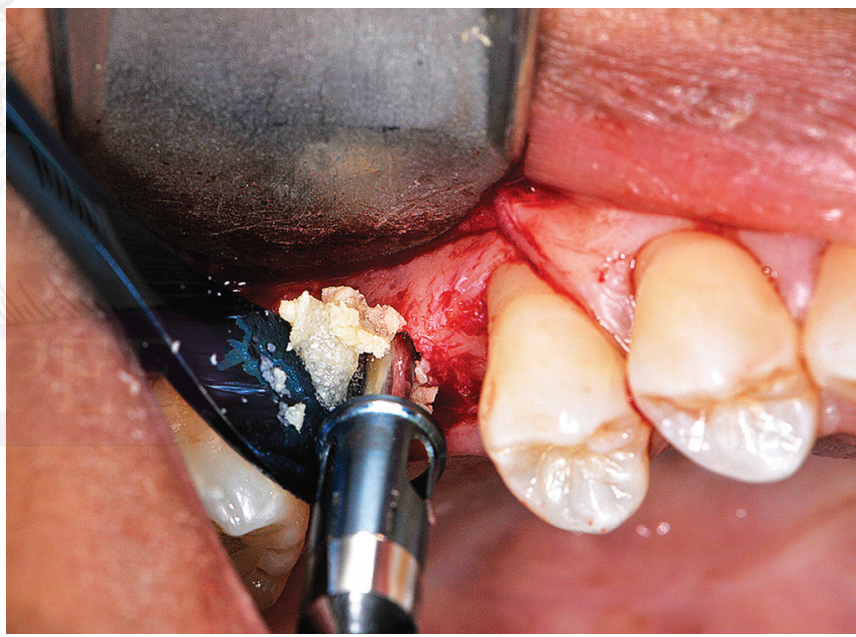

Fig. 13: Graft material is introduced into the osteotomy with the bone packer and a 9-mm stopper

increasing its firmness, but provides some slipperiness to aid in placing it through the osteotomy into the elevated sinus space (Fig. 11D).

Various augmentation materials may be used in both crestal and lateral sinus augmentation procedures. Autogenous blood concentrates such as platelet rich plasma (PRP) have been reported in the literature as improving graft organization and bone development compared to the use of osseous graft materials alone. ${ }^{10-15}$ A 5-mm stopper was placed on the $2.2 \mathrm{~mm}$ end of the bone packer instrument (Fig. 12). PRP fibrin pieces were placed into the osteotomy (Fig. 13) and the bone packer was used to compress the graft into the elevated sinus (Fig. 14). This process with additional pieces of PRP fibrin was repeated until the instrument 


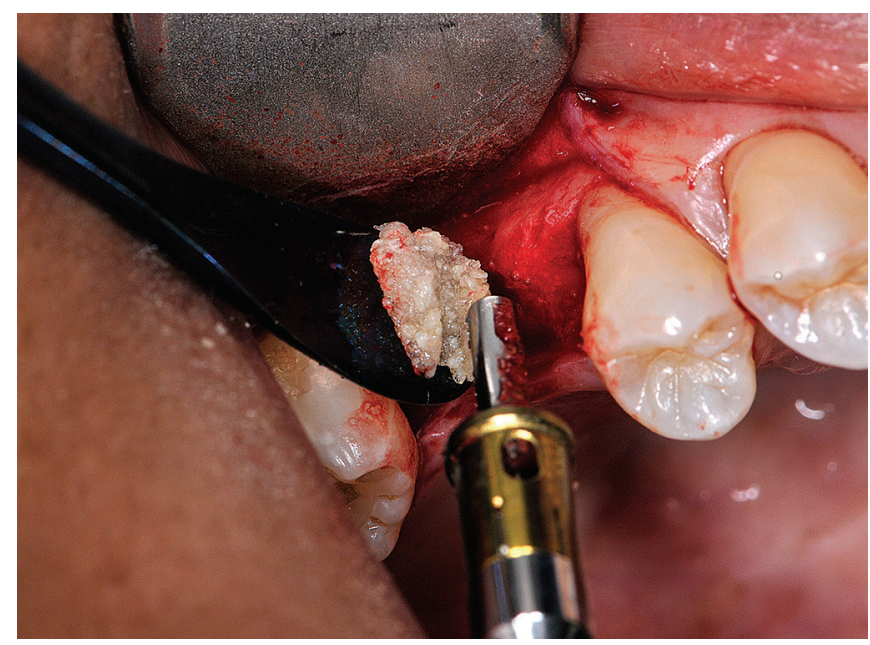

Fig. 14: Additional graft material is introduced into the osteotomy with an 8-mm stopper on the bone packer to fill the elevated sinus with graft

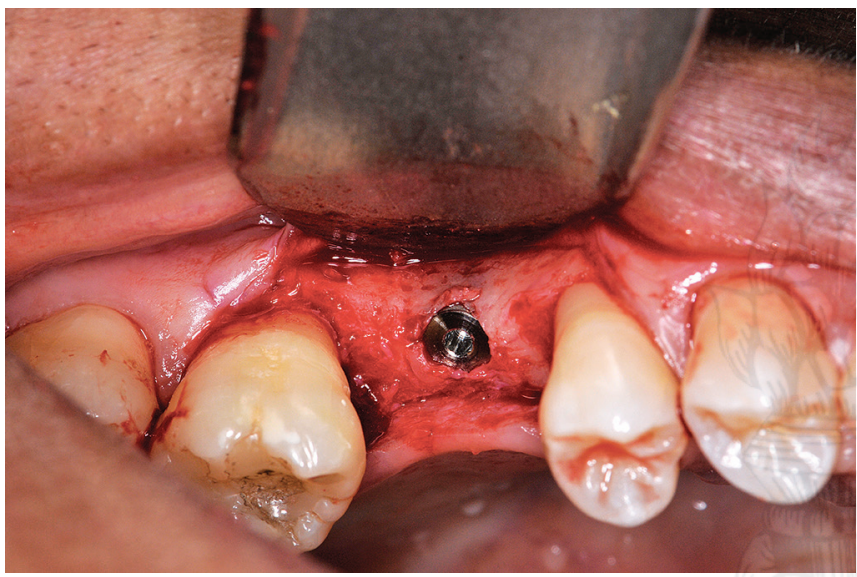

Fig. 16: Implant has been fully seated in relation to the crestal bone

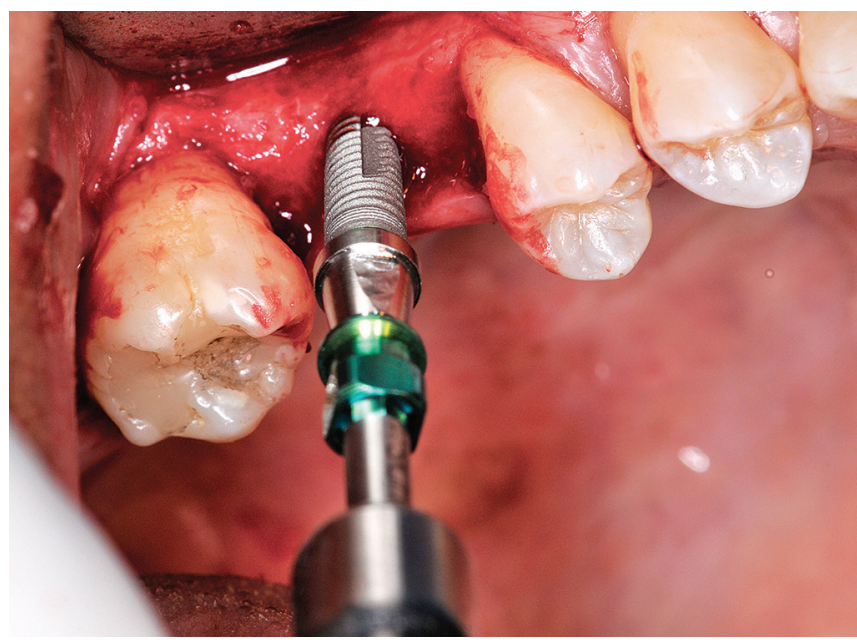

Fig. 15: Implant is introduced into the crestal sinus elevation osteotomy

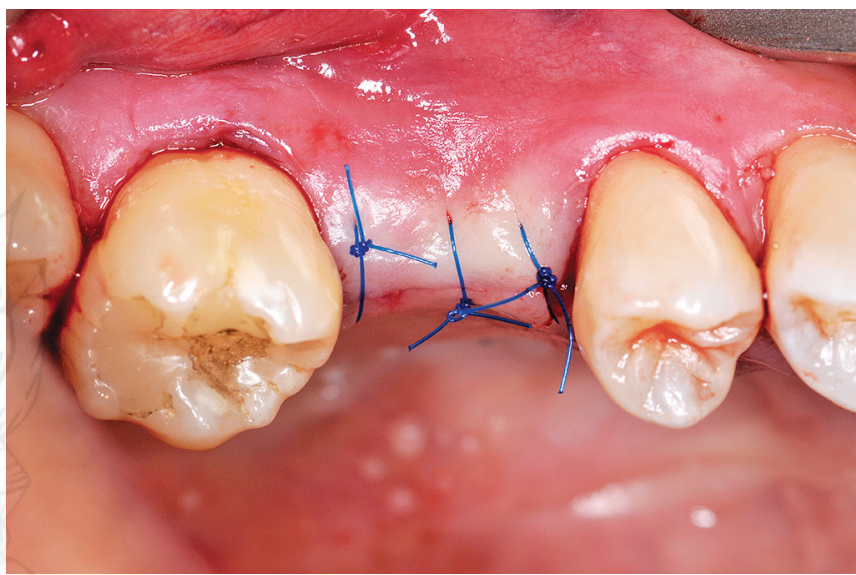

Fig. 17: Flap is repositioned to achieve primary closure and secured with sutures
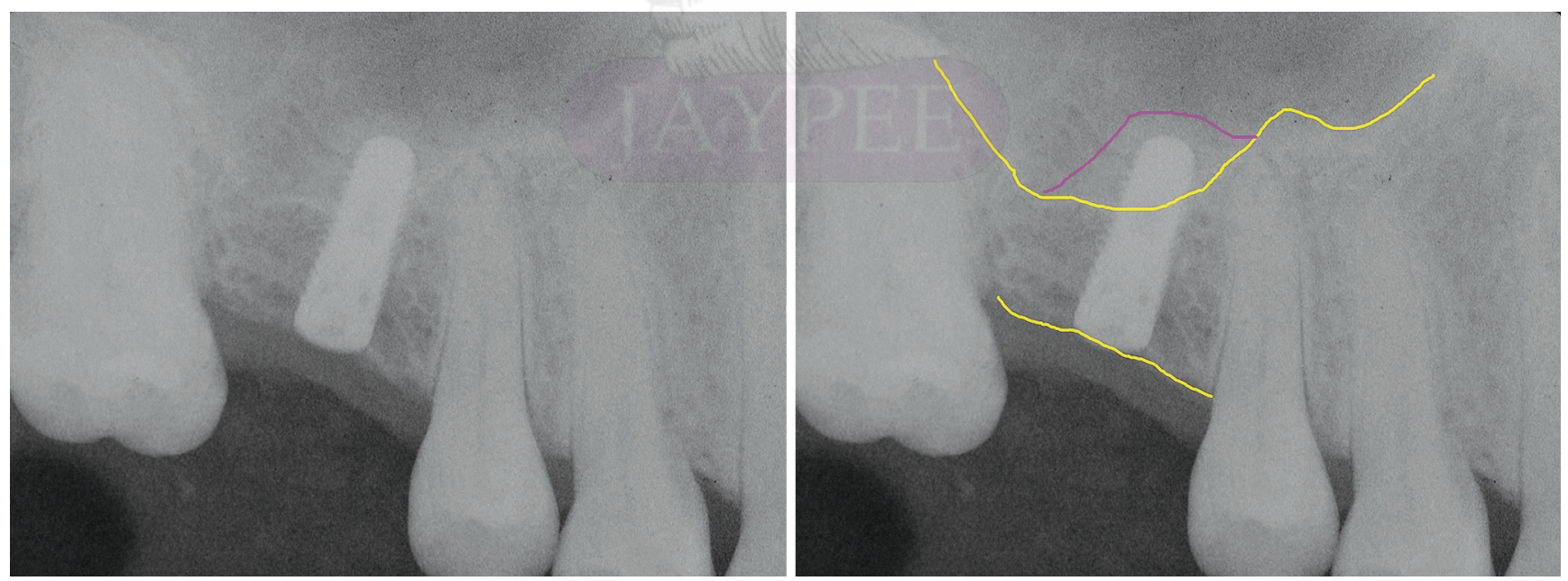

Fig. 18: Radiograph demonstrating the implant placed in the site with crestal elevation to accommodate the implant that was longer than the available crestal height

met resistance and the stopper was close to contact with the crest. This would indicate that the elevated area was filled with a graft material and no further material was needed.

An implant (ImplantVision) of $3.7 \mathrm{~mm} \times 11.5 \mathrm{~mm}$ was placed into the osteotomy using the surgical handpiece (Fig. 15). The implant was placed to be flush with the crest (Fig. 16). The flap was repositioned to achieve primary closure and secured with sutures (Fig. 17). A periapical radiograph was taken to document the implant position, depth, and relation of the apical portion of the implant and elevated sinus graft around it (Fig. 18). 


\section{Conclusion}

Crestal sinus augmentation may be required to allow implant placement when sufficient crestal height is present to stabilize the implant placed simultaneously at the surgical appointment. This approach is less complicated than lateral sinus augmentation procedures with less patient postoperative issues. The illustrated kit and technique improve the clinical outcome with a decrease in membrane tearing. Those practitioners who are routinely placing single or two adjacent implants in the posterior maxilla should be prepared for the need for increasing crestal bone height through a crestal sinus approach.

\section{References}

1. Sharan A, Madjar D. Maxillary sinus pneumatization following extractions: a radiographic study. Int J Oral Maxillofac Implants 2008 Jan-Feb;23(1):48-56.

2. Boyne PJ, James RA. Grafting of the maxillary sinus floor with autogenous marrow and bone. J Oral Surg 1980 Aug;38(8):613-616.

3. Summers RB. A new concept in maxillary implant surgery: the osteotome technique. Compendium 1994 Feb;15(2):152, 154-156, 158 passim; quiz 162.

4. Checchi L, Felice $P$, et al. Crestal sinus lift for implant rehabilitation: a randomised clinical trial comparing the Cosci and the Summers techniques. A preliminary report on complications and patient preference. Eur J Oral Implantol 2010 Autumn;3(3):221-232.

5. Esposito M, Cannizzaro G, et al. Cosci versus Summers technique for crestal sinus lift: 3-year results from a randomised controlled trial. Eur J Oral Implantol 2014 Summer;7(2):129-137.

6. Zitzmann NU, Schärer P. Sinus elevation procedures in the resorbed posterior maxilla. Comparison of the crestal and lateral approaches. Oral Surg Oral Med Oral Pathol Oral Radiol Endod 1998 Jan;85(1):8-17. DOI: 10.1016/S1079-2104(98)90391-2.
7. Peleg M, Mazor Z, et al. Augmentation grafting of the maxillary sinus and simultaneous implant placement in patients with 3 to $5 \mathrm{~mm}$ of residual alveolar bone height. Int J Oral Maxillofac Implants 1999 Jul-Aug;14(4):549-556.

8. Baumann A, Ewers R. Minimally invasive sinus lift. Limits and possibilities in the atrophic maxilla. Mund Kiefer Gesichtschir 1999 May; 3(Suppl 1):S70-S73. DOI: 10.1007/PL00014522.

9. Agamy EM, Niedermeier W. Indirect sinus floor elevation for osseointegrated prostheses. A 10-year prospective study. J Oral Implantol 2010;36(2):113-121. DOI: 10.1563/AAID-JOI-D-09-00085.

10. Messora MR, Nagata MJ, et al. Bone healing in critical-size defects treated with platelet-rich plasma activated by two different methods. A histologic and histometric study in rat calvaria. J Periodontal Res 2008 Dec;43(6):723-729. DOI: 10.1111/j.1600-0765.2008.01084.x, Epub 2008 Aug 14.

11. Mariano R, Messora $M$, et al. Bone healing in critical-size defects treated with platelet-rich plasma: a histologic and histometric study in the calvaria of diabetic rat. Oral Surg Oral Med Oral Pathol Oral Radiol Endod 2010 Jan;109(1):72-78. DOI: 10.1016/j.tripleo.2009.08.003, Epub 2009 Nov 17.

12. Shiu HT, Goss B, et al. Formation of blood clot on biomaterial implants influences bone healing. Tissue Eng Part B Rev 2014 Dec;20(6): 697-712. DOI: 10.1089/ten.teb.2013.0709, Epub 2014 Jul 22.

13. Taschieri S, Karanxha L, et al. Minimally-invasive osteotome sinus floor elevation combined with short implants and platelet-rich plasma for edentulous atrophic posterior maxilla: a five-year follow-up prospective study. J Biol Regul Homeost Agents 2018 Jul-Aug; 32(4):1015-1020.

14. Bae JH, Kim YK, et al. Effects of platelet-rich plasma on sinus bone graft: meta-analysis. J Periodontol 2011 May;82(5):660-667. DOI: 10.1902/jop.2010.100529, Epub 2010 Nov 23.

15. Del Fabbro M, Corbella S, et al. Plasma Rich in Growth Factors Improves Patients' Postoperative Quality of Life in Maxillary SinusFloor Augmentation: Preliminary Results of a Randomized Clinical Study. Clin Implant Dent Relat Res 2015 Aug;17(4):708-716. DOI: 10.1111/cid.12171, Epub 2013 Nov 12. 